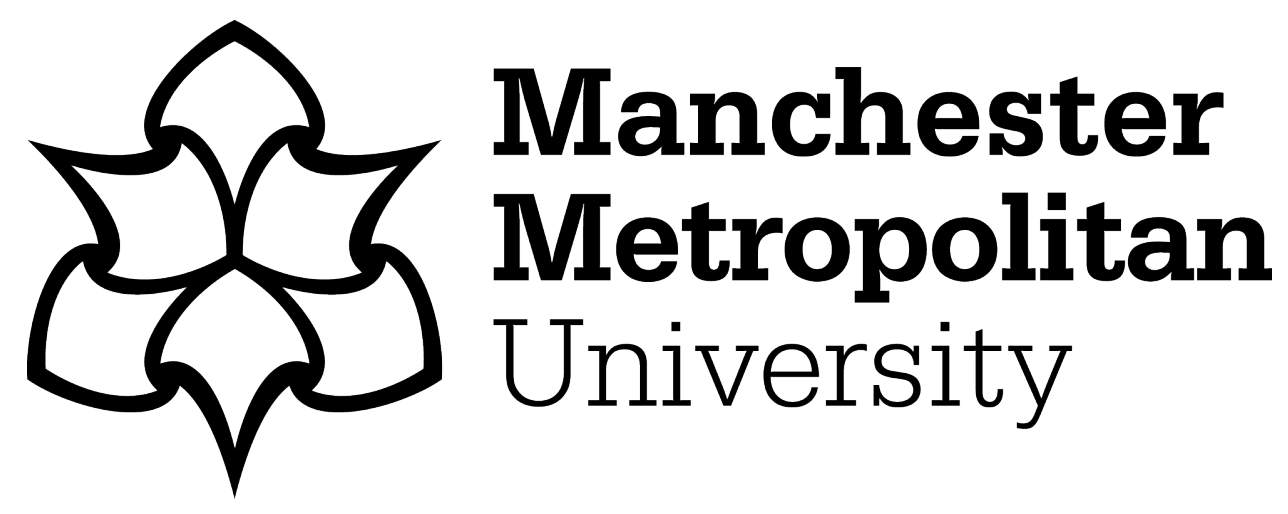

Parry, EJ, Best, JM and Banks, CE (2020) Three-dimensional (3D) scanning and additive manufacturing (AM) allows the fabrication of customised crutch grips. Materials Today Communications, 25.

Downloaded from: https://e-space.mmu.ac.uk/626169/

Version: Accepted Version

Publisher: Elsevier

DOI: https://doi.org/10.1016/j.mtcomm.2020.101225

Usage rights: Creative Commons: Attribution-Noncommercial-No Derivative Works 4.0

Please cite the published version 


\section{Three Dimensional (3D) Scanning and Additive Manufacturing (AM) allows the Fabrication of Customised Crutch Grips}

Elen J. Parry, Joshua M. Best and Craig E. Banks*

Faculty of Science and Engineering, Manchester Metropolitan University, Chester Street, Manchester M1 5GD, UK.

*Correspondence: (C.E.B.): c.banks@mmu.ac.uk; Tel: ++(0)1612471196 (for proofs and correspondence)

This paper investigates the viability of using digital technologies, including 3D laser scanning, reverse engineering techniques and additive manufacturing in order to create bespoke, on demand, customised accessories through re-usable materials to enforce a circular design approach. Crutch users with arthritis are identified as a patient user group who's needs are not met by current solutions.

We identified a remote data collection workflow to capture hand grip profiles, followed by a digital workflow resulting in an additively manufacturable customised product. Threedimensional laser scanning technology captured digital grip profiles which were manipulated in computer aided design (CAD) software to produce a customised hand grip digital file. The prototype was fabricated using Fused Filament Fabrication technology.

We demonstrate that fused filament fabrication, a type of additive manufacturing technology, is an appropriate fabrication method to provide a bespoke and rapidly manufactured low-cost solution; the total timeframe to complete digital scanning and file creation is approximately 20 minutes. Additive manufacturing can be leveraged $24 / 7$ with the customised hand grip rapidly fabricated within $\sim 10$ hours, with only material costs of $£ 4.92 / \sim$ US\$6.15/ €5.65. To our knowledge, this study is the first to discuss using fused filament fabrication to fabricate customised daily living aids.

Highlights: Fused Filament Fabrication combined with three-dimensional scanning provides a low-cost solution for customised daily living aids. Utilising additive manufacturing creates unexplored opportunities for advanced customisation.

Keywords: Additive Manufacturing (3D Printing); 3D scanning; assistive devices; mobility aid; Fused Filament Fabrication (FFF); medical interventions; daily living aids. 


\section{Introduction}

We are an aging population growing in diversity, and this demographic shift has quickly gained awareness, along with the desire to create more inclusive design solutions [1]. The effects of ageing mean many of us are more likely to require daily living aids (DLA) and assistive products to meet the demands of normal every-day life. Although DLA could be essential in increasing or maintaining a good quality of life for many people, abandonment of such products is common practice and is frequently discussed in literature [2]. It is difficult to identify specific reasons for abandonment due to the vast range of devices, and many variations of the same device, without mentioning the complex requirements of each individual user. Generally, device abandonment is believed to relate to user needs being unmet [3], whether that is physically, aesthetically or socially. Inclusive design is an approach which ensures products are accessible to the widest possible range of people [4]. However, this is not always easy, and in many cases, one size does not fit all.

Where user needs are complex, and are likely to vary over time, a modular approach to design can be more effective. Through utilising digital technologies, bespoke products and customised services are becoming an affordable reality. Additive manufacturing (AM) facilitates a production line of one, meaning the manufacture of a batch of identical parts is almost equal in efficiency to a batch of varied parts. Customised earbuds are one example of an on-demand, additively manufactured consumer product [5]. Through combining customisable components with generic products, the scope for better performing products is inevitable.

Daily living aids (DLA) is an area that often incurs design exclusions in relation to products in the marketplace. People with arthritis are often forced to resort to purchasing ergonomic substitutes for standard products that are unsuitable to grip due to design exclusions and the common requirement of the user to be particularly dextrous. In this paper, a workflow for creating bespoke, on demand and customised accessories was formulated in order to meet the needs of crutch users with arthritis in the hand and wrist. A methodology consisting of the remote collection of user data was combined with a digital workflow to demonstrate a quick, low cost solution which caters for the specific needs of each user.

Osteoarthritis (OA) and Rheumatoid Arthritis (RA) are the two most dominant forms of arthritis in the UK, where a total of 8.75 million people have sought treatment for OA [6], and approximately 400,000 adults have RA in UK [7]. This market is continually growing as the number of people with arthritis is expected to double to 17 million by 2030 [8] which indicates a growth of approximately 485,000 annually [7][8]; 12,000 new cases of RA are reported every year [7]. Hence, a total of approximately 497,000 people are diagnosed with RA or OA annually. Off the shelf products available online and through mobility shops do not offer tailored solutions to meet the needs of people suffering from 
arthritis. Crutches are one such item that suffer design exclusions due to the highly economical method of manufacture. Consequently, for people suffering from arthritis, interacting with standard crutches is uncomfortable for a variety of reasons relating to manual dexterity, grip and impact during use [6].

We believe that when products require a high degree of flexibility, creating a modular accessory will be a lower cost and more accessible method of innovation than creating multiple different versions of the same DLA. The longevity of DLA has also been considered. User needs are complex and progressive, often placing different demands on DLA. With the ability to iterate quickly and cost effectively, the proposed digital workflow enables the technical solution to consistently adapt to the ever-changing needs of the end users [9].

A study on axillary crutches suggests that the palm can experience forces of up to 44.4 percent of the entire body weight [10]. Although crutch use differs significantly between users, the majority are susceptible to pain and discomfort in the hand through weightbearing crutch use, which is unsurprising when a significant proportion of weight is absorbed by the forearm during weightbearing crutch use [11]. These findings relate to general crutch use, meaning those with more complex needs will likely experience intensified symptoms.

An overview of ergonomic grip solutions currently available online and in-stores across the UK can be seen in Table 1. Peta UK ( ${ }^{\circledR}$ Colchester, UK) and Performance Health ( ${ }^{\circledR}$ Nottinghamshire, UK) sell products tailored for arthritis sufferers, although each of them requires the user to purchase a bespoke ergonomic substitute or extension for a particular product. Design exclusions also apply when considering this method of delivery as an online platform, which is generally believed to be less accessible to an elderly population. The Digital Inclusion Review 2018 states that "older people are not only less likely than younger people to go online, but those who use the internet are more likely to be narrower users, carrying out fewer activities" [12]. Hence, this paper seeks to explore how a methodology employing the latest digital technologies may be leveraged to better cater to the needs of individuals who use crutches and that also suffer from arthritis in the hand.

In this paper, we report a low-cost, customisable solution to improve the comfort of crutch users. We test the suitability of utilising $3 \mathrm{D}$ scanning and $A M$ technologies to fabricate custom DLA whilst minimising labour intensity, production time and costs, and improving user experience. 
Table 1: An overview of ergonomic grip solutions currently available and the cost to purchase each - prices obtained Jan 2020

\begin{tabular}{|c|c|c|}
\hline Retailer/ Supplier & Item & Price $(€, \$, €)$ \\
\hline \multirow[t]{3}{*}{ Wicker Mobility UK Website [13] } & Queen Edition Cutlery & f12.25 \\
\hline & & US\$15.83 \\
\hline & & $€ 14.16$ \\
\hline \multirow[t]{3}{*}{ Essential Aids UK Website [14] } & Foam Tubings & f5.99 each \\
\hline & & US\$7.74 \\
\hline & & $€ 6.92$ \\
\hline \multirow[t]{3}{*}{ Performance Health UK Website [15] } & Bendable cutlery set & $£ 34$ \\
\hline & & US\$43.95 \\
\hline & & $€ 39.30$ \\
\hline \multirow[t]{9}{*}{ Peta UK Ltd. Website [16] } & Easi-Grip ${ }^{\circledR}$ knife & £11.95 \\
\hline & & US\$15.45 \\
\hline & & $€ 13.81$ \\
\hline & Easi-Grip ${ }^{\circledR}$ fork & f14.95 \\
\hline & & US\$19.32 \\
\hline & & $€ 17.28$ \\
\hline & Easi-Grip ${ }^{\circledR}$ Trowel/Weeder & £11.95 \\
\hline & & US\$15.45 \\
\hline & & $€ 13.81$ \\
\hline
\end{tabular}




\section{Methods}

Figure 1 demonstrates an overview of the methodology employed within this paper. For clarification of discussion, the methodology has been defined into four separate stages, although crossovers occur especially in the 3D scanning and mesh/T-Spline manipulation stages.

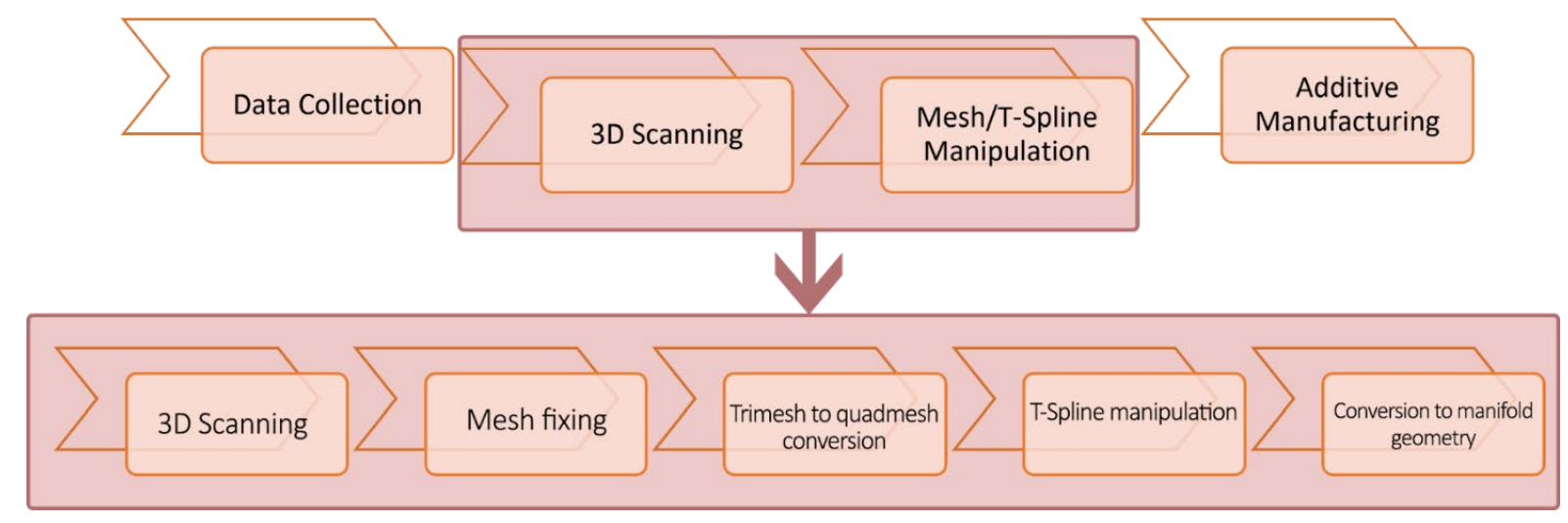

Figure 1: Process Diagram overviews the workflow

\section{Data collection}

Early viability assessments should consider the patient interaction and delivery in order to capture the data required. A viable data collection method and end user interaction is as important as the technical solution. With arthritis sufferers often managing their conditions personally, the parts of their lives that are most affected can differ dramatically person to person. Therefore, complexities in establishing the route to market for a particular technical solution, such as the one proposed, must be considered carefully. To demonstrate proof of concept, a series of hand impressions were provided by the authors using Coolmorph ${ }^{\top M}\left({ }^{\circledR}\right.$ Thermoworx, UK), a thermoplastic (polycaprolactone) which is fully biodegradable and selected due to its non-toxicity and low melting temperature of $42^{\circ} \mathrm{C}$. It is reusable and therefore provides a circular solution for collecting hand profiles, meaning each sample can be reused and reformed which is essential in minimising waste.

\section{D Scanning and mesh workflow}

The representative mould was laser scanned using a ROMER Absolute Arm from Hexagon Manufacturing Intelligence ( ${ }^{\circledR}$ Telford, UK) and Geomagic Wrap scanning software from 3D Systems $\left({ }^{\circledR}\right.$ South Carolina, US). Mesh manipulation processes and digital file creation were carried out in Fusion 360 software and Netfabb in software packages from Autodesk ( ${ }^{\circledR}$ California, US). 


\section{Additive manufacturing}

Prior to Additive Manufacturing (AM) the 3D model must be sliced using dedicated software, which is the process of translating a 3D model into code language. Generically, this code language is referred to as G-code, acting as a set of instructions for the 3D printer to follow. The dedicated software for the chosen 3D printer, an Ultimaker S5 ( ${ }^{\otimes}$ Ultimaker, Netherlands), slices 3D models using Cura ( ${ }^{\circledR}$ Ultimaker, Netherlands), which is a prerequisite for the 3D printing process. This process allows the designer to control specific settings of the print, such as the printing orientation, density and height of each layer among other parameters. Each part can be fine-tuned to best meet its application.

\section{Hardware configuration}

Parameters such as surface quality and build time are impacted by the hardware configuration and resulting print strategy. In this case, a priority is to deliver a custom component quickly. For this reason, a $0.8 \mathrm{~mm}$ print core was selected to extrude Varioshore TPU material. Referring to the objectives of this paper, identifying a digital workflow that minimises labour intensity and production time is key to successfully managing an on-demand service. Therefore, by using a $0.8 \mathrm{~mm}$ print core as opposed to the more commonly used $0.4 \mathrm{~mm}$ print core, the printing time is reduced by $1 \mathrm{hr} 46$ minutes to 9 hrs 23 minutes, with minimal apparent reduction in surface quality.

To increase consistency and printing performance, the Ultimaker S5 should be used with an Air Manager, which is an enclosed inside-out air chamber that sits atop the Ultimaker S5 [17]. This improves printer performance by creating a more controlled environment and stable airflow [18]. Likewise, optimal material performance can be achieved through using Ultimaker's Material Station which is a six-material humidity-controlled storage station. It limits the effects of external influencers that can compromise print quality such as dust and high humidity. Humidity especially can lead to problems with extrusion and a loss of print quality [19]. 


\section{Results}

\section{Data collection}

The thermoplastic moulding process is relatively simplistic. Polymer pellets were placed into hot water $\left(\sim 42^{\circ} \mathrm{C}\right)$ until they turned transparent, at which point they were moulded into a form which loosely represents the crutch handle at half length. This form was placed at the back of the crutch handle (closest to the cylindrical shaft) before following the procedure shown in Figure 2, which captured the grip profile. After 10 minutes, the polymer was removed from the crutch and allowed to harden until cool. This proposed workflow for data collection is demonstrative of a non-invasive, minimal contact interaction that could make a technical solution more accessible to the wider market.

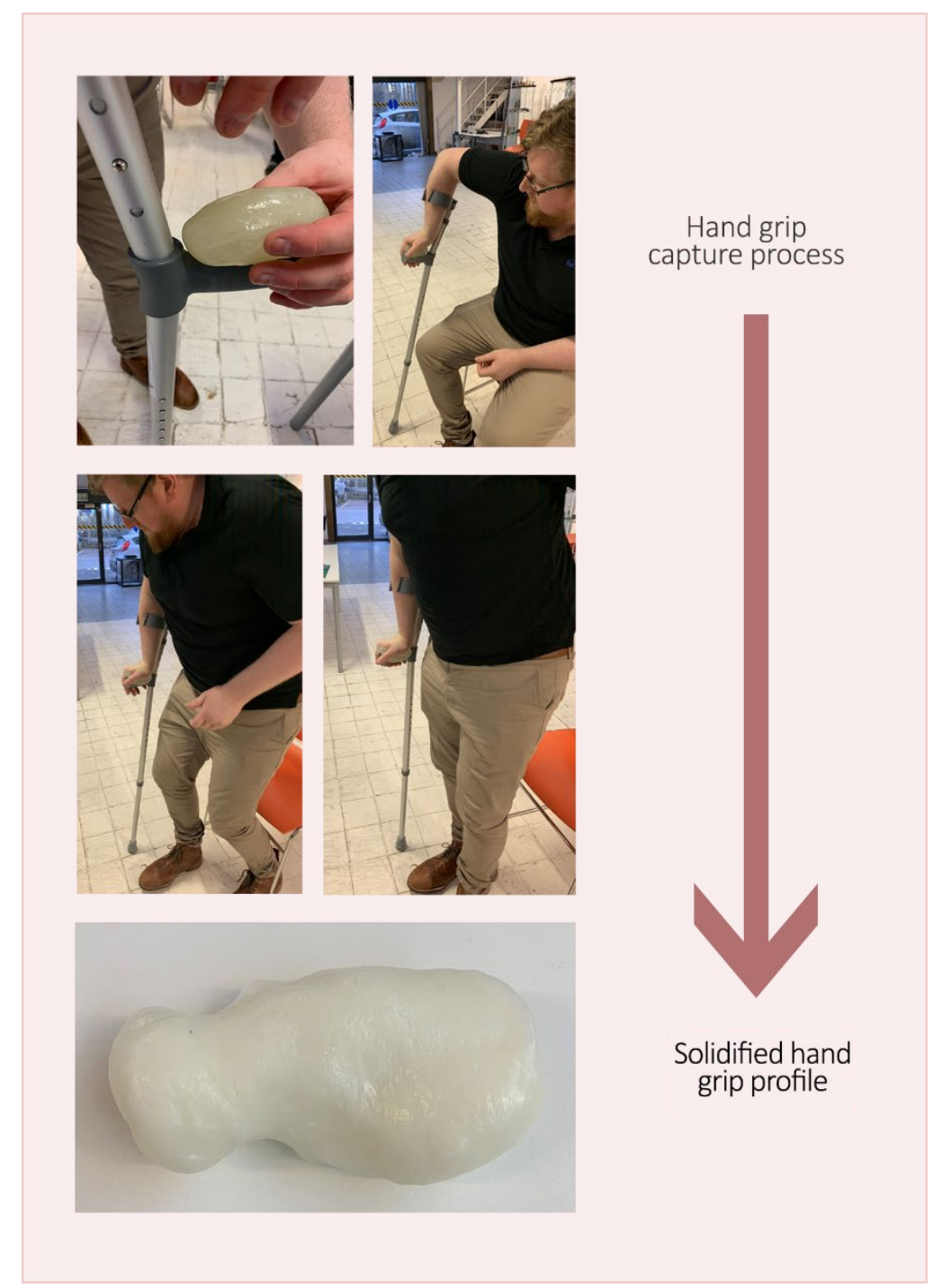

Figure 2: Process images of placing the thermoplastic atop the handle, placing the crutch close to a chair and sitting before using the crutch to support from the seated to standing position and taking three steps forward, resulting in the final solidified hand grip profile 


\section{D Scanning and digital workflow}

3D scanning is a widely used technique for digital data acquisition. Commonly 3D scanners use laser technology, which digitally captures shapes and surfaces often with high precision and speed [20]. Current applications include quality control, reverse engineering and assembly applications to name a few. Democratisation of such technologies has led to a rise in dental, prosthetic and orthotic industries providing customised solutions based on digital scanning. Scanning can be thought of as a less invasive method of quickly collecting personal data as opposed to traditional casting methods. Using a high-quality scanner, a scan can be completed in as little as minutes, making it ideally suited to form part of a workflow aiming to reduce production time.

Work to optimise scanning conditions included ensuring good lighting and mattifying the surface of the provided grip samples. A point cloud was generated through laser scanning which was then converted into a mesh. Mesh cleaning tools used in Geomagic Wrap, including smoothing and filling tools, were used to repair the mesh. This process relaxed the scanned geometry softening any defined lines, which is hoped to increase versatility when used for different activities which deviate from the single movement pattern captured by the grip profile. A Standard Tessellation Language (STL) file was exported from Geomagic Wrap and imported into Fusion 360 which was used to unify, reduce and close the mesh. This type of mesh manipulation generally allows for the creation of smoother and more organic forms.

The modified mesh was converted from a triangular mesh to a quadmesh in Netfabb. This step, which can be seen in Figure 3, is necessary to convert a mesh object into T-Splines, which is a type of geometry used for freeform surface modelling. Within Fusion 360, T-Splines can be manipulated in the sculpt environment, which allows models to be moulded with a virtual toolset. Figure 4 shows selected vertices and edges (in blue and yellow) and a set of arrowed controls for manipulation. This process is comparable to working with clay, only digitally. Without this step, customisation is limited, and the form would replicate that of the hand profile mould. Mesh manipulation is limited, and when considering an unrelated change of form, it can often be unpredictable. Through T-Spline modification the designer has a good amount of aesthetic control, which is an essential element for the success of consumer products.

From a T-spline, Fusion 360 allows conversion into a boundary representation (BRep) of a form. This represents a solid body, which facilitates parametric modelling techniques. From here, a reverse engineered model of a crutch handle was subtracted from the BRep, shown in Figure 5. This removed the material from the inside of the hand grip leaving a void which represents the crutch handle, theoretically creating a perfect fitting cover. 


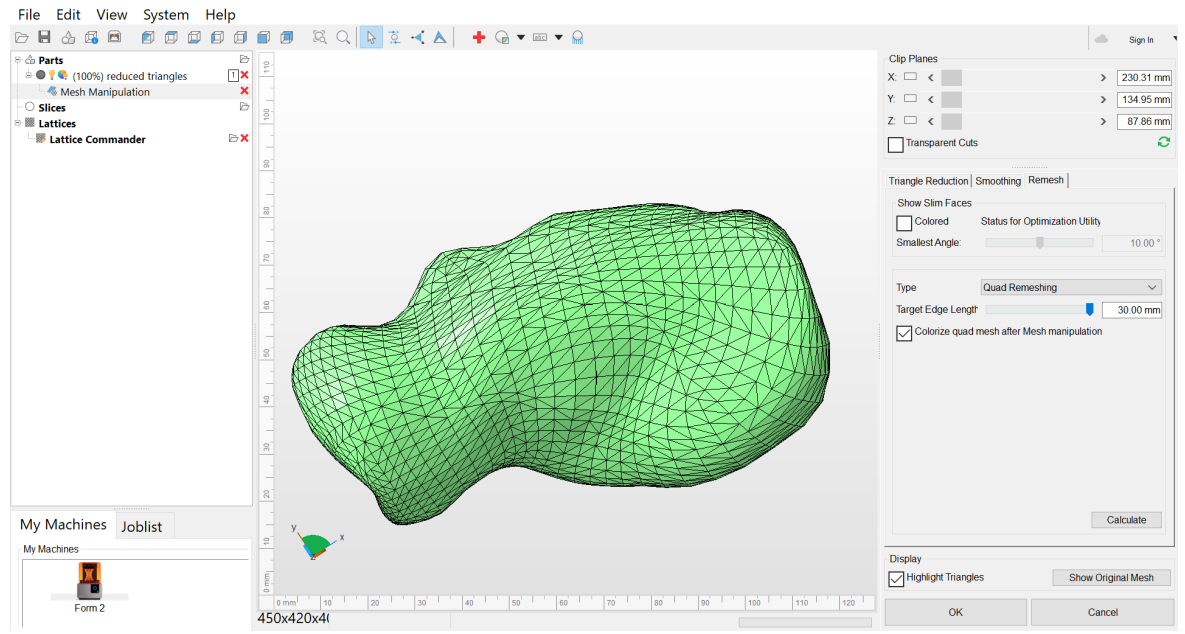

Figure 4: Quad Remeshing workflow actioned in Autodesk Netfabb

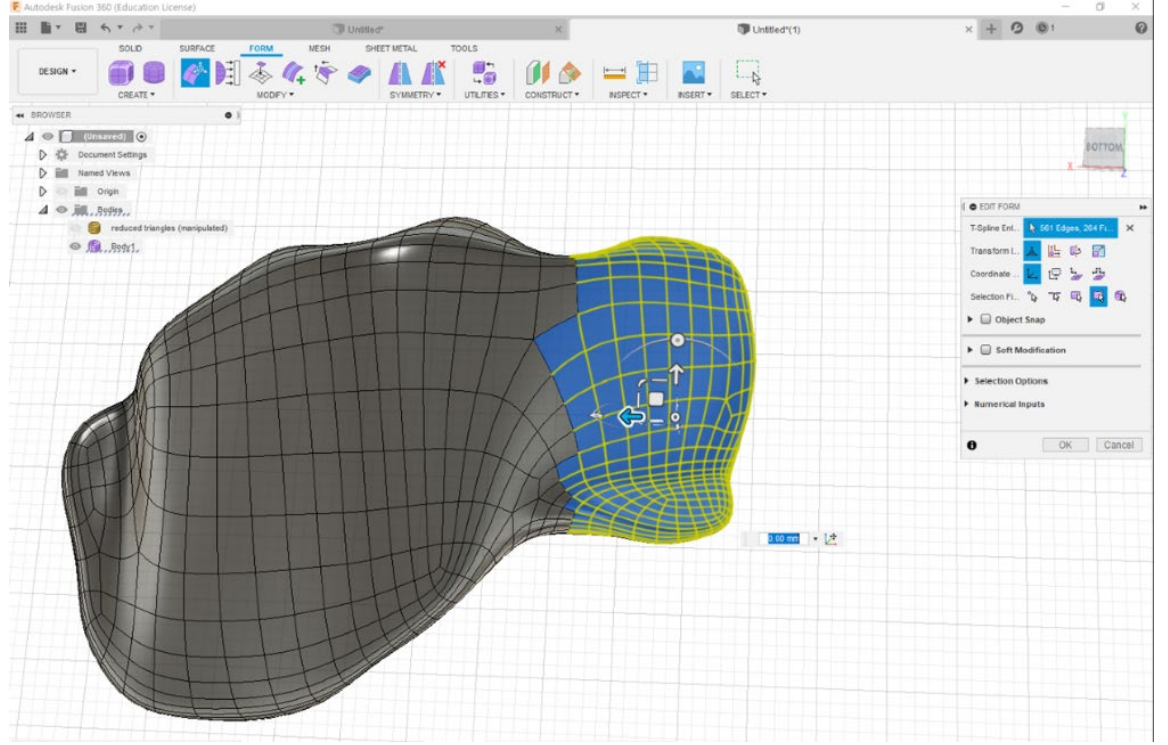

Figure 5: T-spline manipulation, Autodesk Fusion 360

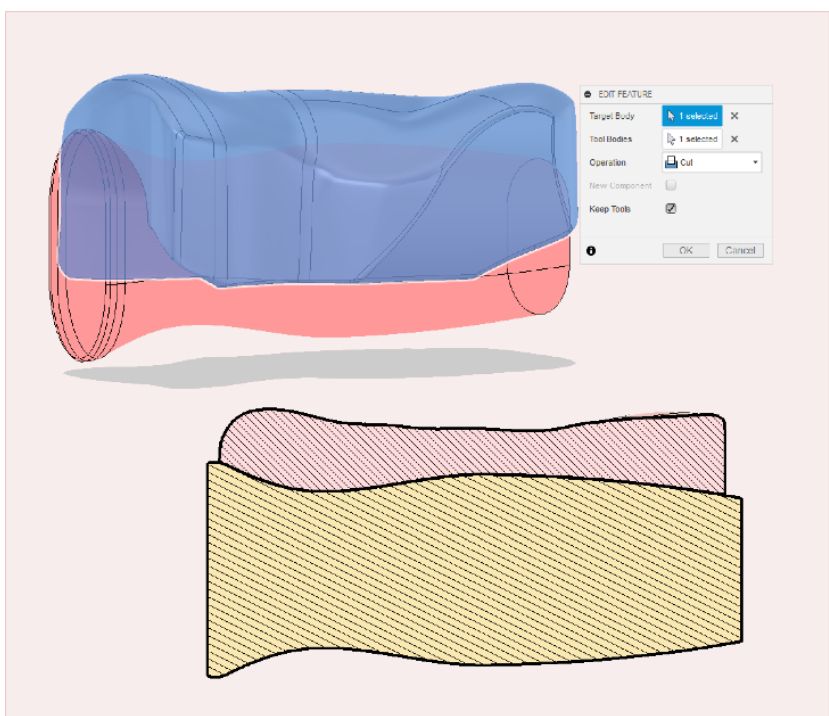

Figure 3: Combine command to remove geometry and a cross-sectional analysis view of the feature in Autodesk Fusion 360 


\section{Material}

A variety of print configurations were tested to determine which yielded the optimum outcome. Through changing the material type, the main variables include the printing temperature and the build plate temperature. The chosen material and printer configuration were validated through several iterations, measured against success criteria seen in figure 6 . This criterion was used to assess multiple print strategies, the most optimal of which was determined to be Varioshore Thermoplastic Polyurethane (TPU) by ColorFabb ( ${ }^{\circledR}$ Belfeld, Netherlands). The density of the material can be varied by adjusting the temperature and material flow rate. An increase in temperature and reduction in flow rate causes acting foaming, reducing the part density. Through iterative experimentation, a set of printing profiles has been determined to deliver variations of density, allowing the creation of a highly customisable accessory.

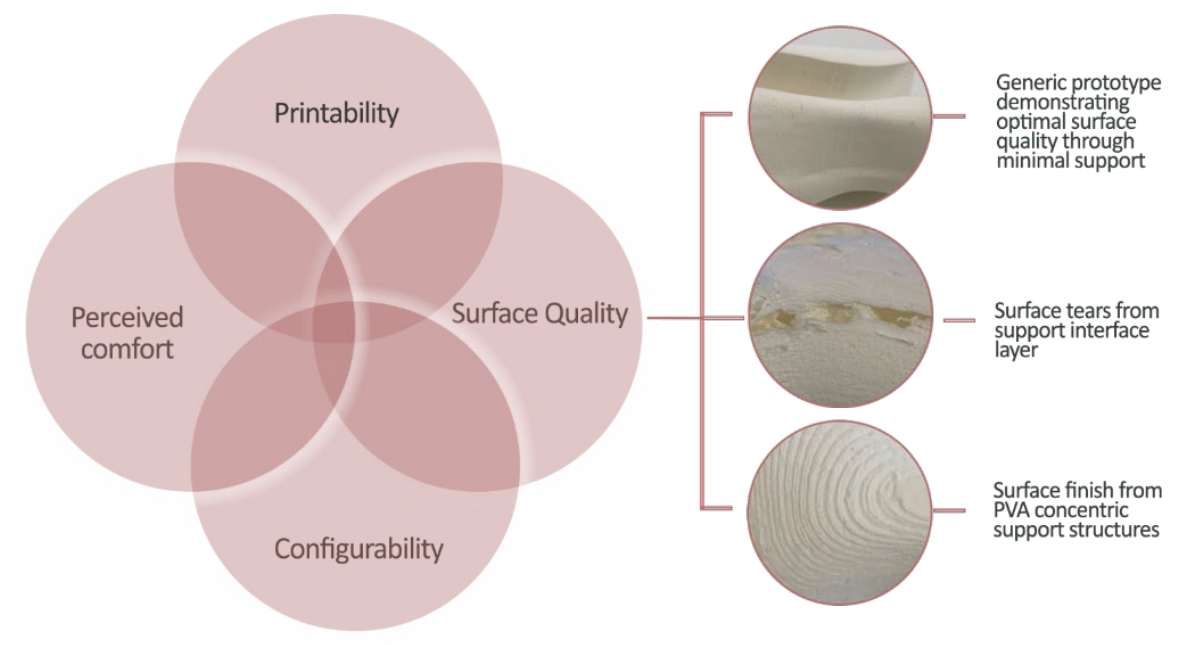

Figure 6: Success criteria decision matrix

\section{Technology}

If configured correctly to meet the success criteria of each part, the results can be low cost and high impact. However, when meeting the requirements for multiple demands, trying to manage compromise can be challenging. For example, the wide range of materials available for FFF printing allow an increased level of flexibility and customisation. This however can cause some compromise in performance and disjoin between manufacturers. Varioshore TPU manufactured by ColorFabb and Breakaway manufactured by Ultimaker were not designed with compatibility in mind for example.

In selecting an appropriate technology and material combination, it was decided that a low cost, accessible and open source hardware such as the Ultimaker $\$ 5$ would provide the most opportunities for iterating suitable solutions. Moreover, with the recent introduction of the Air Manager and Material Station, this desktop printer has ever increasing value for industrial applications. 
In considering materials, Varioshore TPU offers interlayer adhesion that is significantly better than comparable materials. The same interlayer bonding applies to support structures, which is the main reason separating the support materials from the part was difficult. Alternative flexible materials include different variations of TPU. However, an alternative material with similar foaming properties to Varioshore is yet to be identified. Although support removal may be easier with standard TPU, the density and hardness of the part is significantly less controllable. It was suggested that standard TPU prototypes were too firm and would not provide improved comfort. For its soft and light structure, and high degree of customisation, Varioshore was selected as the optimal material for initial proof of concept.

For comparative purposes, the same part was produced using an alternate printing technology, called Stereolithography (SLA), on a Form 2 machine ( ${ }^{\circledR}$ Formlabs, Massachusetts, US) using Elastic Resin ${ }^{\mathrm{TM}}$. The crutch grip, shown in Figure 7, is a fully dense part. Alternative infill structures cannot be specified when setting up the print, therefore structural changes to the 3D model are required for variation, as opposed to the automated infill generation commonly available in FFF slicing software. The decreased ability to vary infill structures on this technology, combined with increased post processing times and greater labour intensity, corroborated the initial strategy regarding open source fused filament printing.

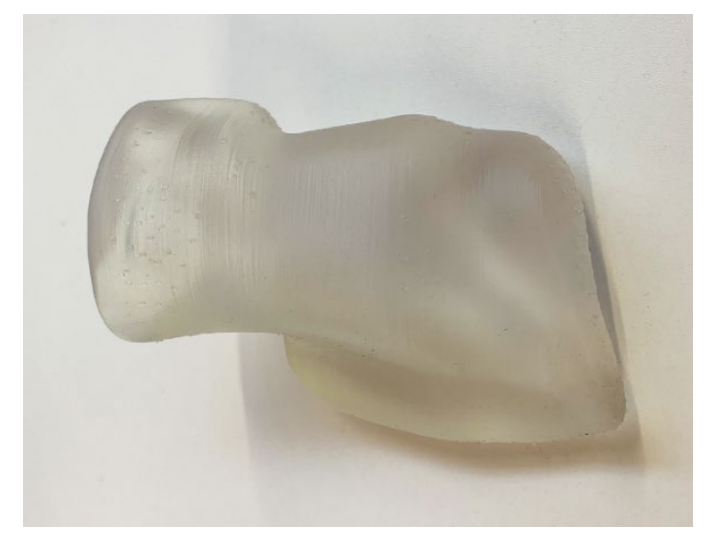

Figure 7: SLA printed grip in elastic resin 


\section{Post-processing}

Sacrificial support material was a necessary addition to enable the part to print using FFF technology, which is a consideration with fused filament printing (Ultimaker, 2016). A number of support configurations were examined in attempting to maintain surface quality whilst minimising post processing requirements, however achieving a consistently satisfactory result was challenging. The issues surrounding support material removal were largely due to the foaming properties of Varioshore TPU.

Minimising support structures through increased spacing resulted in unsupported overhangs. Opposingly, by increasing the amount of support material, excessive bonding between the support structures and the part was observed. Varioshore TPU appeared susceptible to tearing, and whilst removing support material carefully, which was both time consuming and labour intensive, the surface continued to tear shown in Figure 6. A good surface finish is integral to this application. For a functional accessory to add quality for the user, ergonomic comfort should be a priority. Table 2 demonstrates some of the attempted support variations and their effects on surface finish. A previous generic prototype demonstrated an excellent surface finish through minimising support material. This desired surface quality shown in figure 6 is achievable with overhanging geometry which does not exceed 60 degrees on the outer surface. 60 degrees is the maximum critical support angle, meaning the slicing software will only generate support when it is absolutely required. 
Table 2: An overview of three tested support structure techniques, each demonstrated with a digital visual representation of support placement, an image showing the resulting surface finish and comments and observations of each method.

\begin{tabular}{cccc}
\hline Type of support structure & $\begin{array}{l}\text { Visual representation } \\
\text { of support placement }\end{array}$ & Surface finish & Comments \\
\hline
\end{tabular}

\section{Support interface layer}

A dense interface between the model and support material.

Variations include:

- Support roof thickness from $1 \mathrm{~mm}$ to $0.5 \mathrm{~mm}$

- Support roof density from $100 \%$ to $25 \%$
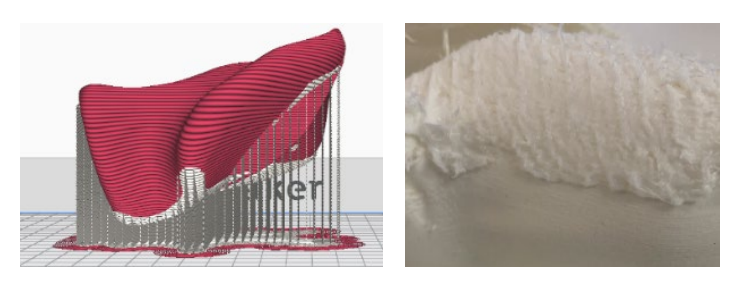

Breakaway support

material has bonded to the Varioshore TPU making it difficult to remove.

The Varioshore part has breakaway support fused into the outer surface which could potentially cause injury or discomfort to the user.

\section{Support pattern variation}

Line distance from $3.5 \mathrm{~mm}$ to $3 \mathrm{~mm}$

- Support interface layer features to reduce the chances of both materials fusing.

- Line infill pattern was selected because generally it is easier to remove than a grid pattern.

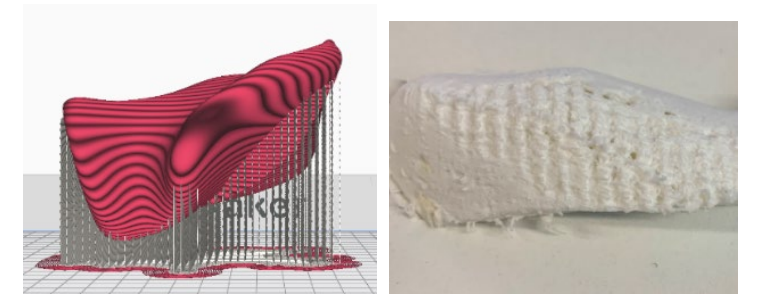

The distance between each support structure is too large.

The foaming properties of Varioshore mean the material is expanding between support struts leaving an uneven surface finish with defined ridges.

\section{Support pattern variation}

Support line distance from $3 \mathrm{~mm}$ to $1 \mathrm{~mm}$

- $\quad$ As a response to the $3 \mathrm{~mm}$ distance above being too wide, the distance has been reduced to $1 \mathrm{~mm}$.

- Reducing the distance between supports could remove the need for an interface layer.
Surface finish remains uneven. Varioshore still expanded between support structures.

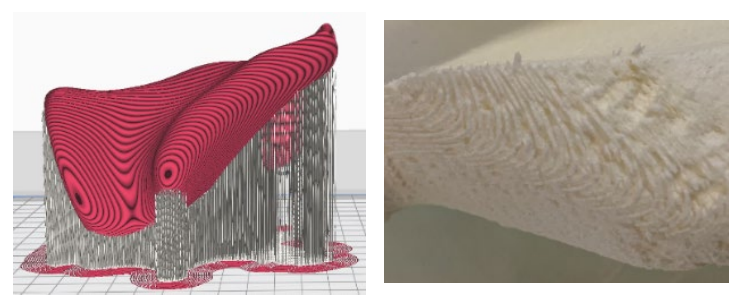

As well as an uneven surface finish, the amount of support material required increased from the previous by $69 \mathrm{~g}$. 
To offer full customisation, the proposed solution should perform with variations of extreme geometry. As an essential requirement of the study, further variations were tested. Polylactic acid is recommended by the manufacturer as a suitable material for generating support structures. When tested, we experienced a similar result to the breakaway support. The best solution came from using Polyvinyl Alcohol (PVA), a water-soluble synthetic polymer which allows for practically labour free support removal. The PVA initially demonstrated poor results, printing with sharp points, leaving an uneven and unpredictable surface finish. Through previous experimentation, adding an interface layer provided a more efficient way to guarantee a smooth surface, the difficulty here was removing the support without tearing the surface. Utilising PVA's soluble properties, an interface layer of $100 \%$ density was selected with a concentric pattern which was dissolved post-print. After dissolving the PVA support material, a satisfactory surface quality was achieved. The PVA concentric support surface quality shown in Figure 6 demonstrates a consistent surface without any unpredicted spikes or holes. With additional testing, we are confident that the surface quality can be increased through further optimising the print strategy.

To aid in discussing the success of the proposed workflow, we have defined a minimal viable product (MVP), which will act as a comparative tool when discussing results. Based on the solutions currently available shown in Table 1, cost is within the region of $£ 30 / \sim$ US\$37.51/ $€ 34.46$ or less which provides a cost-effective solution to DLA. In order to be a potential on-demand service, the turn-around time should ideally align with existing shipping durations of a working week as is typically accepted with most consumer products. This estimate does not consider the time it takes for the user to capture and return their grip profile. The prototype created from the above workflow can be seen in Figures 8 and 9 which was manufactured using FFF technology in Varioshore TPU supported by PVA material. Complete manufacturing time was 10 hrs 5 minutes at a consumable cost of $£ 4.92 / \sim U S \$ 6.15 / \sim 55.65$. When considering overheads and machine depreciation, excluding labour, an expected cost per part to produce would be approximately $£ 9.50 / \sim$ US\$/11.88/ €10.90; this value was quoted by a competitive 3D printing bureau, GoPrint3D ( ${ }^{\oplus}$ Ripon, UK). 


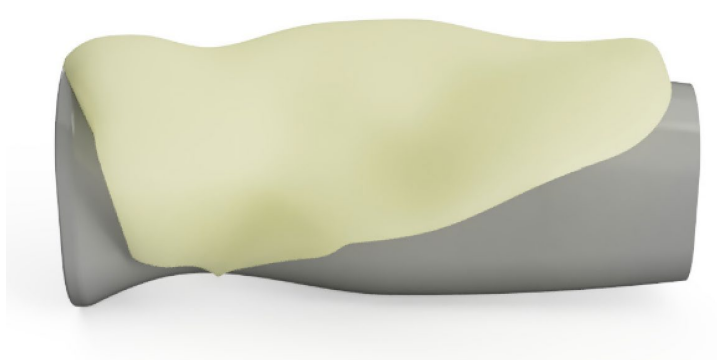

Figure 8: Full customised crutch grip, digital visualisation

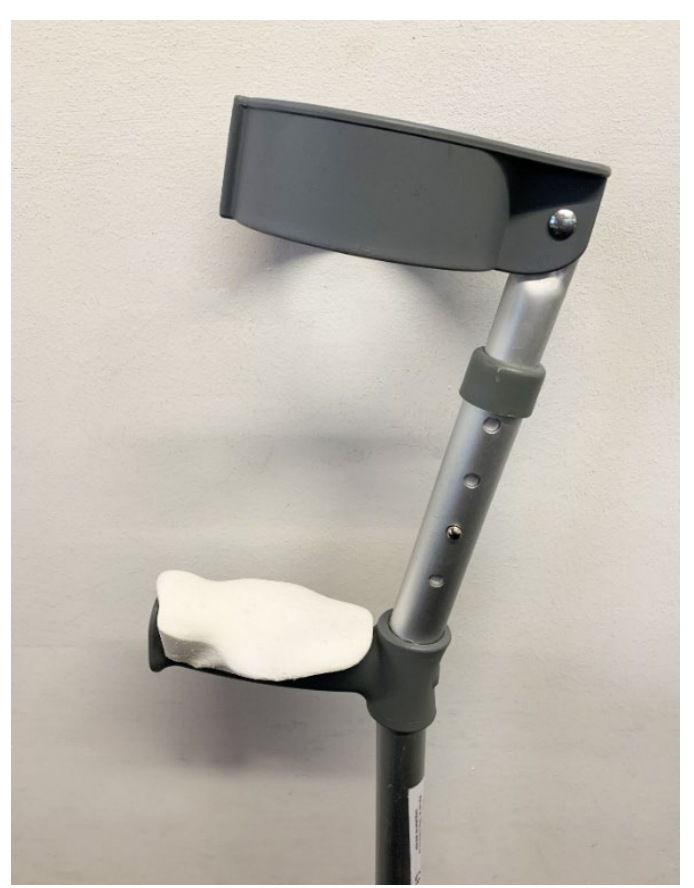

Figure 9: Additively manufactured customised crutch grip installed on a crutch

Note, there is not currently a definitive service which provides users with customised accessories. Customised crutch grips are known to have been manufactured by individuals who have recognised a need, and due to the need not being met, have created their own solution for a customised grip. A method that has been brought to the attention of the authors is hand-crafting customised grips by stitching leather around an internal structure. This, however, is not a widely adopted technique and has been identified by the authors as a singular case. The two most comparable services available are online mobility shops and online craft shops. When considering functionality, users can choose between a range of generic ergonomic hand grips and purchase online. Online craft shops specialise in the delivery of customised products. The customisation, however, is based around aesthetic appearance as opposed to form. Services are available for users to select different colour and fabric handle covers. Although it may increase desirability and acceptance with some users, the functionality is limited as these commonly consist of fabric coated foam tubes. A concise breakdown of the proposed process steps, along with time, cost and labour considerations associated with each step can be seen in Table 3. A lack of service providers in this area has meant a comparison of traditional and proposed workflows is not possible. However, an estimate of what a traditional workflow might look like includes a labour intensive, hand crafting model. 
Table 3: A labour, time and cost breakdown for each process step in the proposed digital workflow.

*This assumes hardware is readily available, prices obtained Jan 2020. Note that there is no comparative workflow for ergonomic grips currently available.

\begin{tabular}{|c|c|c|c|c|}
\hline Process Step & Data Collection & 3D Scanning & $\begin{array}{l}\text { Mesh/T-Spline } \\
\text { Manipulation }\end{array}$ & $\begin{array}{l}\text { Additive } \\
\text { Manufacturing }\end{array}$ \\
\hline $\begin{array}{l}\text { Labour } \\
\text { Intensity }\end{array}$ & $\begin{array}{l}\text { Low, minimal } \\
\text { skill level }\end{array}$ & $\begin{array}{l}\text { Low, semi- } \\
\text { automated data } \\
\text { collection, } \\
\text { minimal skill level }\end{array}$ & $\begin{array}{l}\text { Medium skill } \\
\text { level, semi- } \\
\text { automated data } \\
\text { processing }\end{array}$ & $\begin{array}{l}\text { Low skill level, } \\
\text { automated } \\
\text { production, some } \\
\text { manual post } \\
\text { processing }\end{array}$ \\
\hline $\begin{array}{l}\text { Approximate } \\
\text { Time to } \\
\text { Complete }\end{array}$ & 30 minutes & 10 minutes & 10 minutes & 10 hrs 5 minutes \\
\hline Materials & $£ 2.50$ & $\mathrm{~N} / \mathrm{A}$ & $N / A$ & $£ 4.92$ \\
\hline Cost* & $\begin{array}{l}\sim \text { US\$3.13 } \\
\sim € 2.87 \\
\text { (per 100g) }\end{array}$ & & & $\begin{array}{l}\sim \text { US\$6.15 } \\
\sim € 5.65\end{array}$ \\
\hline
\end{tabular}




\section{Conclusions}

We demonstrate that AM is a viable method of producing customised DLA. A need has been identified to provide a solution offering customised DLA, which is anticipated to improve quality of life for sufferers of arthritis. This proof-of-concept study has identified AM as a low-cost method of quickly producing customised hand grips for crutch users.

Although this study was based around an elbow crutch, the findings are highly transferrable, and the same workflow could be applied to multiple crutch or walking stick variations. With two additional steps applied to the outlined workflow, both the hand profile and crutch profile can be customised. Data would be taken from a laser scan of the crutch handle and would be used in the place of the reverse engineered crutch handle discussed in this study. If a pre-existing CAD model of the intended crutch handle is available, this should be used instead. Through subtracting the crutch handle geometry away from the crutch grip, the customised aid will fit most variations of crutch handles.

We recommend that the MVP in this research area is a solution that is perceived to offer improved comfort when using crutches and that has been produced economically, with minimal end user interaction and minimal manual labour. With this in mind, the proposed workflow has identified strong opportunity for a solution that meets this criterion. This proof-of-concept study will be extended to a user study and reported separately. 


\section{References}

[1] Clarkson J, Coleman R. Inclusive design. Journal of engineering design. 2010;21(2-3):127-9.

[2] Mann WC, Goodall S, Justiss MD, Tomita M. Dissatisfaction and nonuse of assistive devices among frail elders. Assistive technology : the official journal of RESNA. 2002;14(2):130.

[3] Verza R, Carvalho MLL, Battaglia MA, Uccelli MM. An interdisciplinary approach to evaluating the need for assistive technology reduces equipment abandonment. Multiple sclerosis. 2006;12(1):88-93.

[4] Clarkson J, Coleman R. History of Inclusive Design in the UK. Applied Ergonomics. 2015;46:235-47.

[5] Formlabs. 2018. [cited 2020]. Available from: https://formlabs.com/blog/custom-earbudsmanufactured-with-3d-printing/.

[6] Unknown. Osteoarthritis in General Practice. online: Arthritis Research UK; 2013. Available from: https://healthinnovationnetwork.com/resources/osteoarthritis-in-general-practicearthritis-research-uk-2013/

[7] Unknown. (2002) Arthritis: the big picture. Online: Arthritis Research UK; 2002. Available from: https://www.bl.uk/collection-items/arthritis-the-big-picture

[8] Unknown. (2012) Arthritis cases 'set to double to over $17 \mathrm{~m}$ by 2030'. [Online] [Accessed on 12/01/2020] https://www.bbc.co.uk/news/health-17995938

[9] Bogers M, Hadar R, Bilberg A. Additive manufacturing for consumer-centric business models: Implications for supply chains in consumer goods manufacturing. Technological Forecasting \& Social Change. 2016;102:225-39.

[10] Goh JCH, Toh SL, Bose K. Biomechanical study on axillary crutches during single-leg swingthrough gait. Prosthetics and Orthotics International. 1986;10(2):89-95.

[11] Hügle T, Arnieri A, Bünter M, Schären S, Mündermann A. Prospective clinical evaluation of a novel anatomic cuff for forearm crutches in patients with osteoarthritis. BMC musculoskeletal disorders. 2017;18(1):110.

[12] Davidson S. Digital Inclusion Evidence Review 2018. online: Age Uk; 2018.

[13] Shop WM. Queens Left Angled Spoon online2020 [Available from: https://www.wickermobilityshop.co.uk/queens-left-angled-spoon-214-p.asp.

[14] Essentialaids online 2020 [Available from: https://www.essentialaids.com/thick-plastazotefoam-tubing.html.

[15] Sure Grip Bendable Cutlery online2019 [Available from: https://www.performancehealth.co.uk/sure-grip-bendable-cutlery\#sin=126698.

[16] PETA. Innovating for Independence 2020 [Available from: https://peta-uk.com/.

[17] GoPrint3D. Ultimaker S5 Air Manager online2019 [Available from: https://www.goprint3d.co.uk/ultimaker-s5-air-manager.html.

[18] Davies S. Ultimaker reveals S5 advancements and launches S3 3D printing platform at TCT show. TCT. 2019 24/9.

[19] Unknown. online: filament2print. 2018. [cited 2020]. Available from: https://filament2print.com/gb/blog/45 humidity-problems-3d-filaments.html.

[20] Lee SY, Majid Z, Setan H. 3D Data Acquisition For Indoor Assets Using Terrestrial Laser Scanning. ISPRS Annals of Photogrammetry, Remote Sensing and Spatial Information Sciences. 2013;II-2/W1:221-6. 